\title{
Evaluación de la función ejecutiva en una población escolar con síntomas de déficit de atención e hiperactividad
}

\author{
A. Vélez-van-Meerbeke*, I.P. Zamora, G. Guzmán, B. Figueroa, C.A. López Cabra y \\ C. Talero-Gutiérrez
}

Grupo de Investigación en Neurociencias, Escuela de Medicina y Ciencias de la Salud, Universidad del Rosario, Bogotá, Colombia

Recibido el 5 de marzo de 2012; aceptado el 30 de junio de 2012

Accesible en línea el 18 de agosto de 2012

\author{
PALABRAS CLAVE \\ Atención; \\ Trastorno por déficit \\ de atención e \\ hiperactividad; \\ Funciones ejecutivas; \\ Sistema de \\ evaluación de la \\ conducta en niños; \\ Evaluación \\ neuropsicológica \\ infantil; \\ Weschler inteligence \\ scale for \\ children-revised
}

\begin{abstract}
Resumen
Objetivo: : Determinar las alteraciones de las funciones ejecutivas (FE) en niños con trastorno por déficit de atención e hiperactividad (TDAH).

Sujetos y métodos: Se realizó un estudio de casos y controles con una muestra de estudiantes de colegios de Bogotá, Colombia, pertenecientes a los estratos socioeconómicos bajos. El diagnóstico de TDAH se realizó con la lista de chequeo del DSM IV y la escala multidimensional de BASC. Se descartaron los niños que presentaban trastornos cognitivos. Se evaluó el desempeño en 6 medidas de funciones ejecutivas. Se realizaron un análisis bivariado entre variables, un estudio multivariado controlado por sexo y edad, y una regresión logística condicional.

Resultados: Se estudió a 119 niños con síntomas de TDAH y 85 controles con edades comprendidas entre 6 y 13 años. Cuando se controlaron por sexo, edad y tipo de colegio, los niños con TDAH tuvieron un mayor compromiso que los controles en las medidas de FE correspondientes a fluidez verbal y gráfica, figura compleja de Rey-Osterrieth y flexibilidad cognitiva. Cuando se compararon los subgrupos de TDAH, no hubo diferencias entre el grupo mixto con el general. Los casos con inatención sola e hiperactividad-impulsividad sola presentaron dificultades en fluidez gráfica.

Conclusiones: Los niños con síntomas de TDAH presentan mayores problemas en medidas de las FE especialmente en planeación, inhibición, memoria de trabajo y control cognitivo. Parece existir posiblemente una heterogeneidad entre el trastorno de las FE respecto del sexo y la edad.

(c) 2012 Sociedad Española de Neurología. Publicado por Elsevier España, S.L. Todos los derechos reservados.
\end{abstract}

\footnotetext{
* Autor para correspondencia.

Correo electrónico: alberto.velez@urosario.edu.co (A. Vélez-van-Meerbeke).
}

0213-4853/\$ - see front matter @ 2012 Sociedad Española de Neurología. Publicado por Elsevier España, S.L. Todos los derechos reservados. doi:10.1016/j.nrl.2012.06.011 


\section{KEY WORDS}

Attention;

Attention deficit

hyperactivity

disorder;

Executive functions;

Behavioral

assessment system

for children;

Neuropsychological

assessment of

children;

Weschler inteligence

scale for

children-revised
Evaluating executive function in schoolchildren with symptoms of attention deficit hyperactivity disorder

\begin{abstract}
Objective: To identify impairment of executive functions (EF) in children with attention deficit hyperactivity disorder (ADHD).

Subjects and methods: A case-control study was performed on a sample of schoolchildren with low socioeconomic levels in Bogota, Colombia. ADHD was diagnosed using the DSM IV checklist and the Behavior Assessment System for Children scale. Children with cognitive deficits were excluded. We evaluated scores from six measurements of executive function (EF). We conducted a bivariate statistical analysis to compare the variables, a multivariate study controlled by sex and age, and a logistic regression analysis.

Results: The study sample included 119 children with ADHD and 85 controls, all aged between 6 and 12 years. Controlling by sex, age, and type of school showed that EF measurements in children with ADHD were significantly more impaired than in controls, especially for measurements of verbal and graphic fluency, Rey-Osterrieth Complex Figure, and cognitive flexibility. Comparison of ADHD subgroups showed that results in children with multiple deficits were similar to those in the global ADHD group. Graphic fluency impairment was the sole impairment in cases with only attention deficit or only hyperactivity-impulsivity manifestations.

Conclusions: EF measures in children with ADHD revealed more problems, particularly those having to do within planning, inhibition, working memory and cognitive control. Age and sex may affect the degree of EF impairment.

(c) 2012 Sociedad Española de Neurología. Published by Elsevier España, S.L. All rights reserved.
\end{abstract}

\section{Introducción}

La atención es la capacidad de seleccionar los estímulos que son relevantes para el individuo en una situación específica; depende de la maduración de las estructuras y de los sistemas funcionales que permiten el estado de alerta y la filtración de la información en el sistema nervioso central y, por lo tanto, es parte importante de los dispositivos básicos para el aprendizaje ${ }^{1}$. Por otra parte, las funciones ejecutivas (FE) se definen como la capacidad de planear, dirigir, orientar, guiar, coordinar y ordenar la acción conjunta de los elementos para lograr un fin o una meta ${ }^{2}$.

El trastorno por déficit de atención e hiperactividad (TDAH) es una entidad nosológica de alta prevalencia y morbilidad que constituye la enfermedad crónica más frecuente del periodo escolar y representa un problema complejo debido a que aparece en edades tempranas, repercute en la vida diaria del niño y existe la probabilidad de que persista a lo largo de la vida ${ }^{3-5}$. Muchos estudios han puesto en evidencia la relación existente entre el TDAH y el desempeño de las $\mathrm{FE}^{6-8}$. En este sentido, el niño con déficit de atención presenta dificultad para organizar su conducta motora y cognitiva, lo que se hace evidente por la incapacidad de obedecer órdenes y tener un tiempo de espera antes de realizar las acciones y tareas las que finalmente ejecuta en forma desordenada e impulsiva9-11.

Desde el punto de vista etiológico, la tendencia se dirige principalmente al estudio de los receptores de dopamina y, en general, de las vías encargadas del metabolismo de las catecolaminas, las cuales se pueden ver alteradas tanto en el TDAH como en el mantenimiento de algunas $\mathrm{FE}^{12}$.

Cabe destacar que el TDAH puede coexistir con diversos trastornos, como alteraciones del aprendizaje, de la conducta o del estado de ánimo, y con el síndrome de Gilles de la Tourette, entre otros, y el trastorno de las FE de estos niños se presenta aún en ausencia de estas comorbilidades ${ }^{13-15}$.
El objetivo del presente trabajo fue determinar la relación entre el TDAH y el desempeño en las FE en una población escolar de Bogotá.

\section{Sujetos y métodos}

Se realizó un estudio observacional analítico de casos y controles, continuación de una investigación de corte transversal para determinar la prevalencia de enfermedades neuropediátricas en niños de estratos 1 a 4 en colegios públicos y privados de la ciudad de Bogotá. Se seleccionó a los niños que tuvieron síntomas positivos para el TDAH según la lista de chequeo del DSM IV validada para Colombia ${ }^{16}$ y algunos otros niños que mediante el uso de encuestas a padres y maestros fueron sospechosos de presentar el trastorno. El diagnóstico fue comprobado mediante la aplicación de la escala BASC (Behavior Assessment System for Children) ${ }^{17}$. Teniendo en cuenta que se evidenció una baja concordancia en las calificaciones de padres y maestros, se decidió tomar por separado los resultados de las escalas de atención e hiperactividad de padres o de maestros para la conformación final del grupo de casos. Los niños seleccionados tenían que cumplir los siguientes requisitos:

- Niño escolar con lista de chequeo aplicada a padres con $\geq 6$ criterios de los 9 para inatención y un percentil $\geq 85$ en el dominio de atención en la escala de atención BASC aplicada a padres o maestros o

- Niño escolar con lista de chequeo aplicada a padres con $\geq 6$ criterios de los 9 para hiperactividad y un percentil $\geq$ 85 en el dominio de hiperactividad en la escala de BASC aplicada a padres o maestros.

A todos los casos se les aplicó la prueba WISC-R (Weschler inteligence scale for children-revised) para descartar a 
Tabla 1 Variables demográficas

\begin{tabular}{|c|c|c|c|}
\hline Variable & Casos & Controles & $\mathrm{p}$ \\
\hline$N .^{\circ}$ & 119 & 85 & 0,017 \\
\hline Edad & $\begin{array}{l}8,5 \text { años } \\
\text { IC del } 95 \% \\
(8,1-8,9)\end{array}$ & $\begin{array}{l}9,6 \text { años } \\
\text { IC del } 95 \% \\
(9,2-10,1)\end{array}$ & 0,001 \\
\hline \multicolumn{4}{|l|}{ Sexo } \\
\hline Femenino & $25(21,3 \%)$ & $31(36,5 \%)$ & 0,423 \\
\hline Masculino & 94 (79\%) & $54(63,5 \%)$ & 0,002 \\
\hline \multicolumn{4}{|l|}{ Tipo de colegio } \\
\hline Público & 41 & 5 & $<0,001$ \\
\hline Privado & 78 & 80 & 0,874 \\
\hline \multicolumn{4}{|l|}{ Curso } \\
\hline Primero & 33 & 7 & $<0,001$ \\
\hline Segundo & 27 & 9 & 0,002 \\
\hline Tercero & 15 & 19 & 0,384 \\
\hline Cuarto & 17 & 22 & 0,423 \\
\hline Quinto & 13 & 15 & 0,705 \\
\hline Sexto o más & 14 & 13 & 0,847 \\
\hline
\end{tabular}

aquellos que podrían tener alteraciones cognitivas (se utilizó un punto de corte de $\mathrm{Cl}<70$ ). Para el grupo control se evaluó a niños considerados como «normales» de los mismos cursos de los anteriores pero que no cumplían los criterios para TDAH según el DSM IV y el BASC.

Para la evaluación de las FE, se seleccionaron y aplicaron, tanto a sujetos como a controles, subpruebas de la Evaluación Neuropsicológica Infantil (ENI) que valoran la capacidad de programar, estructurar y desarrollar diferentes tipos de tareas que involucran habilidades relacionadas con aspectos semánticos, manejo de vocabulario, capacidad de representación del mismo, copia de figuras o elaboración sin un modelo pero con directriz, así como la capacidad de establecer criterios de clasificación y de cambiar de criterio ante la sugerencia del examinador ${ }^{18}$.

El procesamiento de la información se realizó con los paquetes estadísticos SPSS versión 19.0 para Windows. Para asegurar la calidad de los datos, previamente se hizo una capacitación a todos los investigadores. Durante el trabajo de campo se insistió en la importancia del diligenciamiento completo del formato y por lo menos uno de los investigadores estuvo disponible para resolver las dudas que surgieron. Durante el proceso de digitación se tomó una muestra aleatoria del $10 \%$ de los registros y se hizo una depuración de la base de datos hasta verificar que no existieran inconsistencias.

Para el análisis descriptivo de las variables de tipo cualitativo se utilizaron distribuciones de frecuencia y porcentuales, y en las variables de tipo cuantitativo, medidas de tendencia central y medidas de variabilidad y sus respectivos coeficientes de variación para medir la homogeneidad de los datos. Se evaluó la relación entre los hallazgos de las FE y el TDAH mediante la prueba de asociación de la ji al cuadrado sin corregir de Pearson. Con los datos que mostraron valores de probabilidad $<0,20$, se realizó un estudio de regresión logística condicional o un estudio multivariado (MANCOVA).

Este fue un estudio sin riesgo que siguió los lineamientos jurídicos y éticos del país, y los contemplados en la última modificación de la Declaración de Helsinki de la Asociación Médica Mundial. La participación de las familias fue voluntaria; todos los padres y las madres de los niños firmaron un consentimiento informado y los niños dieron su asentimiento. El estudio fue aprobado por el comité de ética en investigación de la Universidad del Rosario. A todos los niños se les entregó un informe de los resultados. Para quienes obtuvieron puntajes en el $\mathrm{Cl}$ inferiores a 70 en las pruebas psicológicas, se dio una asesoría a los padres para orientarlos sobre el manejo adecuado.

\section{Resultados}

Se obtuvo firma del consentimiento en 250 niños. De estos, se excluyó a 35 por datos incompletos o no concluyentes de TDAH, uno por ser menor de 6 años y 10 por tener un $\mathrm{Cl}$ inferior a 70. La muestra final estuvo conformada por 119 casos y 85 controles, de los cuales 148 fueron hombres y 56 mujeres $(2,6: 1)$ (tabla 1$)$. La relación entre casos y controles en hombres muestra una diferencia significativa a favor con los casos $(p=0,002)$, pero no en las mujeres.

Ciento siete casos pudieron ser clasificados en los subgrupos del TDAH: 35 presentaron inatención $(29,9 \%), 11$ hiperactividad/impulsividad $(9,4 \%)$ y 63 trastorno mixto $(52,9 \%) ; 10$ casos no pudieron ser calificados por resultados incongruentes. Se estudió a niños de todos los cursos de primaria, pero se evidenció una diferencia de casos frente a los controles en los cursos más tempranos, lo que no representó problemas en el momento de realizar los análisis.

Le edad media \pm desviación estándar fue de $9 \pm 2,26$ años (6-15). Se encontró que los controles eran en promedio 1,11 $(0,49-1,72)$ años mayores que los casos $(p<0,001)$ (tabla 1$)$. En la distribución por edad de la muestra se encontró que los controles presentan una curva normal $(p=0,13)$ mientras que en los casos se aprecia una desviación hacia los niños más pequeños $(p=0,006)$. 
Tabla 2 Puntajes en la prueba de WISC

\begin{tabular}{|c|c|c|c|}
\hline Variable & Media & Desviación estándar & $\mathrm{p}$ \\
\hline \multicolumn{4}{|c|}{ Razonamiento perceptual } \\
\hline Total & 108,57 & 15,11 & \multirow{3}{*}{0,051} \\
\hline Control & 113,05 & 15,22 & \\
\hline Casos & 107,09 & 14,84 & \\
\hline \multicolumn{4}{|c|}{ Comprensión verbal } \\
\hline Total & 85,63 & 12,37 & \multirow{3}{*}{0,400} \\
\hline Control & 87,11 & 13,82 & \\
\hline Casos & 85,15 & 11,88 & \\
\hline \multicolumn{4}{|c|}{ Velocidad de procesamiento } \\
\hline Total & 90,97 & 12,51 & \multirow{3}{*}{0,680} \\
\hline Control & 93,89 & 13,03 & \\
\hline Casos & 90,00 & 12,24 & \\
\hline \multicolumn{4}{|c|}{ Memoria de trabajo } \\
\hline Total & 88,69 & 11,98 & \multirow{3}{*}{0,267} \\
\hline Control & 90,74 & 12,71 & \\
\hline Casos & 88,02 & 11,71 & \\
\hline \multicolumn{4}{|l|}{ Cl total } \\
\hline Total & 92,10 & 12,68 & \multirow{3}{*}{0,077} \\
\hline Control & 95,26 & 13,11 & \\
\hline Casos & 91,08 & 12,42 & \\
\hline
\end{tabular}

En el grupo incluido, el puntaje del WISC fue de 92,1 , lo que se catalogó dentro del promedio. Se evidenció que los casos presentaron puntajes menores que los controles pero sin encontrar diferencias significativas (tabla 2).

Se encontró una disminución de los percentiles de las calificaciones de las pruebas de FE en los casos, salvo en la fluidez verbal semántica (animales). Esta diferencia fue significativa en fluidez verbal semántica (frutas) y fonémica, fluidez gráfica no semántica, dibujo de la figura de Rey, planeación e interpretación (tiempo de ejecución [segundos]) para realizar el diseño correcto), flexibilidad cognitiva (incapacidad para mantener la organización y respuestas perseverativas) y dibujo de la figura humana (tabla 3).

Al categorizar los percentiles de las FE evaluadas en mayores o menores del percentil < 10 (extremo anormal), se encontraron hallazgos significativos en las siguientes: fluidez verbal fonémica $(p=0,044)$; fluidez gráfica no semántica $(p=0,05)$, fluidez gráfica total $(p=0,04)$ y la incapacidad para mantener la organización $(p=0,01)$.

Cuando se revisan los diferentes grupos, se encuentra que los niños del grupo de inatención pura presentan mayores dificultades en la fluidez gráfica no semántica $(p=0,05)$ y fluidez gráfica total $(p=0,01)$. En aquellos con hiperactividad pura, el único componente significativo fue la fluidez gráfica total $(p=0,03)$. En el grupo mixto, se encontraron alteraciones en la fluidez gráfica no semántica y total $(p=0,038$ y $p=0,042)$, en la ejecución inicial de la figura de Rey $(p=0,04)$ y en la flexibilidad cognitiva $(p=0,018)$.

Cuando se evaluaron los grupos por sexo, se evidenció que los hombres presentaron diferencias significativas en la ejecución inicial de la figura de Rey $(p<0,001)$ y el porcentaje de respuestas perseverativas $(p=0,037)$; las mujeres con TDAH tuvieron diferencias respecto del grupo control en fluidez verbal fonémica $(p=0,040)$, fluidez gráfica no semántica $(p=0,036)$, ejecución inicial $(p=0,002)$ y recobro $(0,050)$ de la figura de Rey, dibujo de la figura humana $(p=0,000)$, en diferentes aspectos de la flexibilidad cognoscitiva, como en la perseveración de las respuestas $(p=0,000)$ y en la incapacidad para mantener la organización $(p=0,000)$. Al comparar hombres y mujeres entre sí, no se encontraron diferencias significativas en el desempeño.

El análisis multivariado (MANCOVA) de las FE agrupadas y controladas por edad y tipo de colegio no mostró diferencias significativas entre los casos con TDAH y los controles.

$\mathrm{El}$ análisis de regresión logística demostró que las FE relacionadas con planeación y organización, memoria de trabajo, fluidez gráfica e inhibición fueron las más comprometidas en pacientes con síntomas de TDAH (Cox and Snell $R$ squared $=26,5$ ) cuando el modelo se controló por edad y tipo de colegio (tabla 4).

\section{Discusión}

Teniendo en cuenta la divergencia de la calificación atribuida por padres y maestros en la lista de chequeo del DSM IV y la escala del BASC, y analizando su efecto en la conformación del grupo definitivo, se decidió incluir en la muestra a todos los niños que tuvieran una calificación positiva en ambas escalas, independientemente de quién lo catalogara con síntomas de TDAH. Los investigadores son conscientes de que esta medida disminuye la homogeneidad del grupo y es posible que se haya incluido a algunos niños que tengan síntomas en situaciones particulares o que tengan cuadros en los que el trastorno de atención o hiperactividad sea secundario. Esta diferencia de apreciación de la conducta de los niños entre los padres y los maestros puede deberse a múltiples causas, entre las que se pueden mencionar el nivel de tolerancia y de exigencia frente a la conducta y al rendimiento académico, el volumen de los cursos y aspectos de tipo étnico, cultural y social. Hay que recalcar la asimetría respecto de la edad entre casos y controles en el grupo entre los 6 y los 8 años, lo que corresponde a los primeros años de escolaridad. Estos resultados estarían asociados a aspectos relacionados con maduración, motivación y expectativas de padres y maestros, lo cual ya había sido mencionado en un estudio previo ${ }^{19}$. La mayoría importante de hombres encontrada estuvo en relación con la mayor prevalencia del TDAH en los hombres respecto de las mujeres ${ }^{20,21}$.

La prueba de WISC cumplió 2 funciones en este trabajo. La primera, descartar a los niños cuyos resultados se situaran por debajo de lo aceptado como normal en lo que se refiere a habilidades cognoscitivas $(\mathrm{Cl}<70)$ y que pudieran comprometer el rendimiento de los niños en otras pruebas, enmascarando un trastorno de la atención. La segunda, para evaluar el desempeño diferencial entre los casos y los controles en la escala total y en las diferentes subpruebas, algunas de las cuales se asocian a la FE. Aunque no hubo diferencias significativas, se puede apreciar que los niños con síntomas de TDAH tienen promedios inferiores respecto de los niños controles en todas las subescalas. En el estudio de Romero-Ayuso et al., al evaluar algunas subpruebas del WISC en niños con TDAH, los subgrupos de inatención o mixto en comparación con los controles sanos evidenciaron un menor rendimiento en las pruebas relacionadas con 
Tabla 3 Evaluación de las pruebas de función ejecutiva entre los casos de TDAH y los controles

\begin{tabular}{|c|c|c|c|c|}
\hline Subprueba & Media & $\begin{array}{l}\text { Desviación típica } \\
\text { de la media }\end{array}$ & Error estándar & $\mathrm{p}$ \\
\hline \multicolumn{5}{|c|}{ Fluidez verbal semántica (frutas) } \\
\hline Control & 44,80 & 28,521 & 3,112 & \multirow[t]{2}{*}{0,034} \\
\hline Casos & 36,16 & 25,683 & 2,364 & \\
\hline \multicolumn{5}{|c|}{ Fluidez verbal semántica (animales) } \\
\hline Control & 37,67 & 24,807 & 2,707 & \multirow[t]{2}{*}{0,301} \\
\hline Casos & 41,05 & 24,568 & 2,262 & \\
\hline \multicolumn{5}{|c|}{ Fluidez verbal fonémica } \\
\hline Control & 42,33 & 25,721 & 2,806 & \multirow[t]{2}{*}{0,036} \\
\hline Casos & 35,13 & 26,108 & 2,403 & \\
\hline \multicolumn{5}{|c|}{ Fluidez verbal total } \\
\hline Control & 38,98 & 25,896 & 2,826 & \multirow[t]{2}{*}{0,098} \\
\hline Casos & 33,07 & 23,720 & 2,184 & \\
\hline \multicolumn{5}{|c|}{ Fluidez gráfica semántica } \\
\hline Control & 34,86 & 28,358 & 3,094 & \multirow[t]{2}{*}{0,454} \\
\hline Casos & 30,93 & 25,650 & 2,361 & \\
\hline \multicolumn{5}{|c|}{ Fluidez gráfica no semántica } \\
\hline Control & 52,60 & 25,798 & 2,815 & \multirow[t]{2}{*}{0,015} \\
\hline Casos & 43,45 & 27,787 & 2,558 & \\
\hline \multicolumn{5}{|c|}{ Fluidez gráfica total } \\
\hline Control & 41,61 & 27,334 & 2,982 & \multirow[t]{2}{*}{0,073} \\
\hline Casos & 35,05 & 27,217 & 2,506 & \\
\hline \multicolumn{5}{|c|}{ Figura de Rey inicial } \\
\hline Control & 55,16 & 35,902 & 3,917 & \multirow[t]{2}{*}{0,000} \\
\hline Casos & 31,97 & 27,286 & 2,523 & \\
\hline \multicolumn{5}{|c|}{ Figura de Rey recobro } \\
\hline Control & 10,08 & 4,657 & 0,508 & \multirow[t]{2}{*}{0,055} \\
\hline Casos & 9,25 & 2,886 & 0,266 & \\
\hline \multicolumn{5}{|c|}{ Planeación e interpretación ( $n .^{\circ}$ diseños correctos) } \\
\hline Control & 62,87 & 11,027 & 1,203 & \multirow{2}{*}{0,510} \\
\hline Casos & 62,51 & 16,312 & 1,502 & \\
\hline \multicolumn{5}{|c|}{ Planeación e interpretación ( $n .^{\circ}$ movimientos realizados) } \\
\hline Control & 50,54 & 28,694 & 3,131 & \multirow[t]{2}{*}{0,275} \\
\hline Casos & 46,11 & 29,747 & 2,738 & \\
\hline Planeación & liseños $m$ & ento) & & \\
\hline Control & 51,89 & 30,658 & 3,345 & 0,121 \\
\hline Casos & 45,90 & 27,901 & 2,569 & \\
\hline Planeación & iempo de & os] para realizar el & orrecto) & \\
\hline Control & 129,57 & 57,263 & 6,248 & 0,000 \\
\hline Casos & 184,09 & 132,362 & 12,185 & \\
\hline Flexibilidac & ensayos r & & & \\
\hline Control & 43,44 & 31,927 & 3,484 & 0,581 \\
\hline Casos & 35,81 & 24,375 & 2,244 & \\
\hline Flexibilidac & respues & & & \\
\hline Control & 50,62 & 27,670 & 3,019 & 0,848 \\
\hline Casos & 49,50 & 28,618 & 2,635 & \\
\hline Flexibilidac & spuestas & & & \\
\hline Control & 49,00 & 31,390 & 3,425 & 0,092 \\
\hline Casos & 41,98 & 26,702 & 2,458 & \\
\hline
\end{tabular}


Tabla 3 (Continuación)

\begin{tabular}{|c|c|c|c|c|}
\hline Subprueba & Media & $\begin{array}{l}\text { Desviación típica } \\
\text { de la media }\end{array}$ & Error estándar & $\mathrm{p}$ \\
\hline \multicolumn{5}{|c|}{ Flexibilidad cognitiva (total de errores) } \\
\hline Control & 53,29 & 32,648 & 3,562 & \multirow[t]{2}{*}{0,061} \\
\hline Casos & 45,19 & 27,775 & 2,557 & \\
\hline \multicolumn{5}{|c|}{ Flexibilidad cognitiva (\% de errores) } \\
\hline Control & 55,42 & 31,864 & 3,477 & \multirow[t]{2}{*}{0,075} \\
\hline Casos & 48,54 & 27,564 & 2,537 & \\
\hline \multicolumn{5}{|c|}{ Flexibilidad cognitiva ( $n .^{\circ}$ de categorías) } \\
\hline Control & 55,04 & 25,499 & 2,782 & \multirow[t]{2}{*}{0,819} \\
\hline Casos & 53,45 & 26,193 & 2,411 & \\
\hline \multicolumn{5}{|c|}{ Flexibilidad cognitiva (incapacidad para mantener la organización) } \\
\hline Control & 34,02 & 30,758 & 3,356 & \multirow[t]{2}{*}{0,001} \\
\hline Casos & 46,85 & 30,067 & 2,768 & \\
\hline \multicolumn{5}{|c|}{ Flexibilidad cognitiva ( $n .^{\circ}$ de respuestas perseverativas) } \\
\hline Control & 55,80 & 9,220 & 1,006 & \multirow[t]{2}{*}{0,000} \\
\hline Casos & 50,81 & 7,577 & 0,697 & \\
\hline \multicolumn{5}{|c|}{ Flexibilidad cognitiva (\% de respuestas perseverativas) } \\
\hline Control & 57,70 & 10,477 & 1,143 & \multirow[t]{2}{*}{0,000} \\
\hline Casos & 51,31 & 8,562 & 0,788 & \\
\hline \multicolumn{5}{|c|}{ Dibujo de la figura humana } \\
\hline Control & 43,93 & 28,951 & 3,178 & \multirow[t]{2}{*}{0,006} \\
\hline Casos & 31,70 & 20,734 & 1,917 & \\
\hline
\end{tabular}

la memoria de trabajo ${ }^{22}$. Hallazgos similares reportan Lambek et al. al comparar la prueba de retención de dígitos en niños con TDAH comparados con niños normales ${ }^{23}$.

El desempeño en la evaluación de las FE entre los casos y los controles muestra, en general, diferencias, siendo estos últimos más eficientes en sus respuestas. Lambek et al. evidenciaron que el desempeño de los niños con TDAH fue estadísticamente más bajo que los niños sin síntomas de TDAH en todas las pruebas realizadas ${ }^{23}$. Estos mismos resultados fueron encontrados por Shuai en China, que estudió a 375 niños con TDAH y 125 controles con edades comprendidas entre los 6 y los 15 años $^{24}$. En el presente estudio, estas diferencias son significativas, específicamente en algunos tipos de tareas que implican mayor capacidad de planeación y memoria de trabajo. Es el caso de la fluidez verbal, para la que se requiere del recobro del vocabulario conocido, pero estructurado en criterios semánticos y fonémicos, de la fluidez gráfica no semántica, la ejecución de la figura de Rey y la realización del dibujo de la figura humana que implican planeación, atención, memoria de trabajo, programación de la ejecución de diseños y copias complejas, y en aquellas tareas que implican cambios de criterio, procesos inhibitorios y flexibilidad cognitiva. Vale la pena mencionar el trabajo de Lopez Campos, en el cual se encontró que la estructura factorial que mejor explicaba la variancia, tanto en los niños con TDAH como en el grupo control, estaba compuesta por los siguientes factores: categorización, fluidez verbal y atención sostenida y flexibilidad cognitiva, los cuales se encuentran alterados en los pacientes evaluados en el presente estudio ${ }^{25}$. Scheres et al. encontraron que los

Tabla 4 Análisis de regresión logística de las funciones ejecutivas

\begin{tabular}{|c|c|c|c|c|c|c|c|}
\hline \multirow[t]{2}{*}{ Variable } & \multirow[t]{2}{*}{ B } & \multirow[t]{2}{*}{$\mathrm{EE}$} & \multirow[t]{2}{*}{ Wald } & \multirow[t]{2}{*}{ Significación } & \multirow[t]{2}{*}{$\operatorname{Exp}(B)$} & \multicolumn{2}{|c|}{ IC del 95\% para Exp (B) } \\
\hline & & & & & & Inferior & Superior \\
\hline Copia inicial de la figura de Rey & 0,763 & 0,414 & 3,4 & 0,065 & 2,144 & 0,953 & 4,822 \\
\hline Recobro de la figura de Rey & 1,119 & 0,481 & 5,42 & 0,02 & 3,061 & 1,193 & 7,85 \\
\hline $\begin{array}{l}\text { Fluidez cognoscitiva: } \\
\text { incapacidad para mantener la } \\
\text { atención }\end{array}$ & 0,683 & 0,318 & 4,62 & 0,032 & 1,98 & 1,062 & 3,691 \\
\hline Fluidez gráfica total & 1,425 & 0,509 & 7,817 & 0,005 & 4,156 & 1,531 & 11,281 \\
\hline Fluidez gráfica semántica & 0,892 & 0,458 & 3,792 & 0,051 & 2,44 & 0,994 & 5,989 \\
\hline Constante & $-2,151$ & 0,685 & 9,87 & 0,002 & 0,116 & & \\
\hline
\end{tabular}

EE: error estándar; Exp (B): odds ratio. 
niños con TDAH presentaban déficits en las pruebas de control de impulsos, inhibición, planeación y fluidez verbal ${ }^{9}$. Sin embargo, estas diferencias desaparecieron cuando se controlaron por edad y género. Lambek igualmente encontró que no hubo un efecto del género cuando se ajustaba por edad pero sí, al contrario, un efecto de la edad cuando se controlaba por el género ${ }^{23}$. Seidman, entre tanto, evidenció una alteración de las FE en relación con el TDAH independiente de la edad y el género ${ }^{7}$. En este sentido, las FE varían en función de la edad y el género en los niños con TDAH. Sarkis menciona, por ejemplo, que cuando se aplica la prueba de la torre de Londres, a mayor edad, los niños utilizan menos tiempo para planear sus estrategias y realizan menos violaciones, y en relación con el género, los hombres hacen las tareas en un tiempo más corto que las mujeres posiblemente por mayor impulsividad o porque saben solucionar las tareas más rápidamente ${ }^{26}$. En el presente estudio se encontraron algunas diferencias por género cuando se analizó en forma independiente a los pacientes con síntomas de TDAH y los controles; al comparar los casos con TDAH entre sí, la ejecución en las pruebas fue similar entre hombres y mujeres.

Como es habitual en nuestra población y la de otros países, se encontró predominio de los casos con alteración mixta de TDAH en relación con los subgrupos de hiperactividad sola e inatención sola ${ }^{14,27}$. Cuando se analizan estos subgrupos en el desempeño de las FE, se encontró que el grupo mixto presenta resultados similares a los del grupo total. Sin embargo, los subgrupos de inatención sola e hiperactividad sola muestran alteraciones en áreas más específicas, sobre todo en la capacidad de planeación para la ejecución de figuras abstractas (fluidez gráfica). Shaui et al. en China refieren que los subgrupos mixto e inatento presentan alteraciones en múltiples tareas de inhibición, cambio de criterio, fluidez verbal y teoría de la mente, mientras que el subgrupo hiperactivo tiene mayor déficit en tareas relacionadas solamente con la memoria visual y la teoría de la mente ${ }^{24}$. Otros autores no han encontrado diferencias entres los subtipos de TDAH y las medidas de las $\mathrm{FE}^{28,29}$.

Teniendo en cuenta los hallazgos del análisis multivariado, se puede inferir que las funciones que están relacionadas con la planeación y la organización, la memoria de trabajo, la fluidez gráfica y parcialmente la inhibición son las de mayor impacto en los pacientes con síntomas de TDAH.

Las FE son procesos complejos que representan habilidades emergentes relacionadas con la edad y el desarrollo del individuo en el momento de la evaluación. Así mismo están directamente asociadas con los estímulos medioambientales, en los cuales el niño ha estado inmerso desde su nacimiento. No existe, desafortunadamente, claridad ni un estándar para evaluar uniformemente a los niños. Cada autor utiliza diferentes tipos de pruebas de acuerdo con un marco conceptual y se hace muy difícil realizar la comparación entre los hallazgos obtenidos. Existe igualmente una disparidad en los resultados cuando se compara a los niños si no se controlan por edad y sexo. Sin embargo, en el caso de los niños con TDAH, los hallazgos son consistentes en cuanto la presencia de un mayor número de errores en tareas de planeación, inhibición y memoria de trabajo. Estos son factores que podrían estar influyendo en el aprendizaje del niño y tendrían que ser evaluados en el momento de plantear un manejo adecuado. Papazian, en su estudio con prescolares con diagnóstico de TDAH comparados con un grupo control, demostró que un entrenamiento temprano en FE lleva a una disminución de la incidencia e intensidad del déficit de atención y de los trastornos del aprendizaje esperados ${ }^{30}$. Por lo tanto, sería conveniente que los niños con riesgo de presentar TDAH sean evaluados precozmente y se involucre a la familia y al colegio en un plan orientado a desarrollar habilidades ejecutivas.

\section{Financiación}

Este trabajo fue realizado como parte de la línea de investigación en Neurociencia cognitiva y fue financiado en parte por el Fondo de Investigaciones de la Universidad del Rosario y por la Fundación para la Promoción de la Investigación y la Tecnología del Banco de la República.

\section{Conflicto de intereses}

Los autores declaran no tener ningún conflicto de intereses.

\section{Agradecimientos}

Los autores agradecen a la Dra. Magola Delgado, directora de Educación Formal de la Caja de Compensación de Colsubsidio, y a los rectores de los colegios por habernos permitido evaluar a los niños.

\section{Bibliografía}

1. Estévez-Gonzalez A, Garcia-Sanchez C, Junque C. La atención: una compleja función cerebral. Rev Neurol. 1997;25:1989-97.

2. Lopera Restrepo F. Funciones ejecutivas: aspectos clínicos. Rev Neuropsicol Neuropsiq Neurociencias. 2008;8:59-76.

3. Cornejo JW, Osío O, Sánchez Y, Carrizosa J, Sánchez G, Grisales $\mathrm{H}$, et al. Prevalencia del trastorno por déficit de atención-hiperactividad en niños y adolescentes colombianos. Rev Neurol. 2005;40:716-22.

4. Pineda DA, Puerta IC, Merchán V, Arango CP, Galvis AY, Velasquez $B$, et al. Factores perinatales asociados con la aparición del trastorno por deficiencia de atención en niños de la comunidad colombiana «paisa». Rev Neurol. 2003;36:609-13.

5. Biederman J, Petty CR, Evans M, Small J, Faraone SV. How persistent is ADHD? A controlled 10-year follow-up study of boys with ADHD. Psychiatry Res. 2010;177:299-304.

6. Lambek R, Tannock R, Dalsgaard S, Trillingsgaard A, Damm D, Thomsen PH. Executive dysfunction in school-age children with ADHD. J Atten Disord. 2011;15:646-55.

7. Seidman LJ, Biederman J, Monuteaux MC, Valera E, Doyle AE, Faraone SV. Impact of gender and age on executive functioning: do girls and boys with and without attention deficit hyperactivity disorder differ neuropsychologically in preteen and teenage years? Dev Neuropsychol. 2005;27:79-105.

8. Biederman J, Monuteaux MC, Doyle AE, Seidman LJ, Wilens $T E$, Ferrero F, et al. Impact of executive function deficits and attention-deficit/hyperactivity disorder (ADHD) on academic outcomes in children. J Consult Clin Psychol. 2004;72:757-66.

9. Scheres A, Oosterlaan J, Geurts H, Morein-Zamir S, Meiran N, Schut $\mathrm{H}$, et al. Executive functioning in boys with ADHD: primarily an inhibition deficit? Arch Clin Neuropsychol. 2004;19:569-94. 
10. Rosselli M, Jurado Maria B, Matute E. Las funciones ejecutivas a través de la vida. Rev Neuropsicol Neuropsiq Neurociencias. 2008;8:23-46.

11. Biederman J, Petty CR, Doyle AE, Spencer T, Henderson CS, Marion B, et al. Stability of executive function deficits in girls with ADHD: a prospective longitudinal followup study into adolescence. Dev Neuropsychol. 2008;33:44-61.

12. Rebollo MA, Montiel S. Atención y funciones ejecutivas. Rev Neurol. 2006;42(Suppl 2):S3-7.

13. Nass R, Bressman S. Attention deficit hyperactivity disorder and Tourette syndrome: what's the best treatment? Neurology. 2002;58:513-4.

14. Pineda DA, Lopera F, Palacio JD, Ramírez D, Henao GC. Prevalence estimations of attention-deficit/hyperactivity disorder: differential diagnoses and comorbidities in a Colombian sample. Int J Neurosci. 2003;113:49-71.

15. Talero-Gutierrez C, Velez-Van-Meerbeke A, Gonzalez-Reyes R. A clinical study of ADHD symptoms with relation to symptoms of learning disorders in schoolchildren in Bogota, Colombia. J Atten Disord. 2012;16:157-63.

16. Pineda DA, Henao GC, Puerta IC, Mejía SE, Gómez LF, Miranda LM, et al. Uso de un cuestionario breve para el diagnóstico de deficiencia atencional. Rev Neurol. 1999;28:365-72.

17. Pineda DA, Kamphaus RW, Mora O, Puerta IC, Palacio LG, Jimenez I, et al. Uso de una escala multidimensional para padres de niños de 6 a 11 años en el diagnóstico de deficiencia atencional con hiperactividad. Rev Neurol. 1999;28:952-9.

18. Rosselli Cock M, Matute Villasenor E, Ardila Ardila A, Botero Gomez VE, Tangarife Salazar GA, Echevarria Pulido SE, et al. Evaluación Neuropsicológica Infantil (ENI): batería para la evaluación de niños entre 5 y 16 años de edad, Estudio normativo colombiano. Rev Neurol. 2004;38:720-31.

19. Talero-Gutierrez C, Espinosa-bode A, Velez-vanMeerbeke A. Trastorno de atención en las escuelas públicas de una localidad de Bogotá: percepción de los maestros. Rev Fac Med UN Col. 2005;53:212-8.

20. Novik TS, Hervas A, Ralston SJ, Dalsgaard S, Rodrigues Pereira R, Lorenzo MJ. Influence of gender on attentiondeficit/hyperactivity disorder in Europe-ADORE. Eur Child Adolesc Psychiatry. 2006;15(Suppl 1):i15-24.
21. Pineda DA, Lopera F, Henao GC, Palacio JD, Castellanos FX. Confirmación de la alta prevalencia del trastorno por déficit de atención en una comunidad colombiana. Rev Neurol. 2001;32:217-22.

22. Romero-Ayuso DM, Maestu F, Gonzalez-Marques J, RomoBarrientos C, Andrade JM. Disfunción ejecutiva en el trastorno por déficit de atención con hiperactividad en la infancia. Rev Neurol. 2006;42:265-71.

23. Lambek R, Trillingsgaard A, Kadesjo B, Damm D, Thomsen PH. Gender differences on the Five to Fifteen questionnaire in a non-referred sample with inattention and hyperactivityimpulsivity and a clinic-referred sample with hyperkinetic disorder. Scan J Psychol. 2010;51:540-7.

24. Shuai L, Chan RC, Wang Y. Executive function profile of Chinese boys with attention-deficit hyperactivity disorder: different subtypes and comorbidity. Arch Clin Neuropsychol. 2011;26:120-32.

25. Lopez-Campo GX, Gomez-Betancur LA, Aguirre-Acevedo DC, Puerta IC, Pineda DA. Componentes de las pruebas de atención y función ejecutiva en niños con trastorno por déficit de atención/hiperactividad. Rev Neurol. 2005;40:331-9.

26. Sarkis SM, Sarkis EH, Marshall D, Archer J. Self-regulation and inhibition in comorbid ADHD children: an evaluation of executive functions. J Atten Disord. 2005;8:96-108.

27. Vélez van Meerbeke A, Talero-Gutierrez C, González-Reyes RMI. Prevalencia de trastorno por déficit de atención con hiperactividad en estudiantes de escuelas de Bogotá, Colombia. Act Neurol Colomb. 2008;24:6-12.

28. Di Trani M, Casini MP, Capuzzo F, Gentile S, Bianco G, Menghini D, et al. Executive and intellectual functions in attentiondeficit/hyperactivity disorder with and without comorbidity. Brain Dev. 2011;33:462-9.

29. Wodka EL, Mostofsky SH, Prahme C, Gidley Larson JC, Loftis C, Denckla MB, et al. Process examination of executive function in ADHD: sex and subtype effects. Clin Neuropsychol. 2008;22:826-41.

30. Papazian O, Alfonso I, Luzondo RJ, Araguez N. Entrenamiento de la función ejecutiva en prescolares con trastorno por déficit de atención/hiperactividad combinado: estudio prospectivo controlado y aleatorizado. Rev Neurol. 2009;48(Suppl 2):S119-22. 